\title{
Recombinant protein blends: silk beyond natural design
}

Dinjaski Nina ${ }^{1}$, Kaplan David L. ${ }^{1}$

${ }^{1}$ Department of Biomedical Engineering, Tufts University, Medford, MA, 02155, USA

CORRESPONDING AUTHOR *

David L. Kaplan

Department of Biomedical Engineering

Tufts University

4 Colby Street

Medford, MA 02155, USA;

Phone: (617)626-3251;

Fax: (617)627-3231;

David.Kaplan@tufts.edu 


\section{Abstract}

Recombinant DNA technology and new material concepts are shaping future directions in biomaterial science for the design and production of the next-generation biomaterial platforms. Aside from conventionally used synthetic polymers, numerous natural biopolymers (e.g., silk, elastin, collagen, gelatin, alginate, cellulose, keratin, chitin, polyhydroxyalkanoates) have been investigated for properties and manipulation via bioengineering. Genetic engineering provides a path to increase structural and functional complexity of these biopolymers, and thereby expand the catalogue of available biomaterials beyond that which exists in nature. In addition, the integration of experimental approaches with computational modeling to analyze sequence-structure-function relationships is starting to have an impact in the field by establishing predictive frameworks for determining material properties. Herein, we review advances in recombinant DNA-mediated protein production and functionalization approaches, with a focus on hybrids or combinations of proteins; recombinant protein blends or "recombinamers". We highlight the potential biomedical applications of fibrous protein recombinamers, such as Silk-Elastin Like Polypeptides (SELPs) and Silk-Bacterial Collagens (SBCs). We also discuss the possibility for the rationale design of fibrous proteins to build smart, stimuli-responsive biomaterials for diverse applications. We underline current limitations with production systems for these proteins and discuss the main trends in systems/synthetic biology that may improve recombinant fibrous protein design and production.

Key Words: recombinant biomaterials; chimeras; silk-elastin-like polypeptides; biomedical application 


\section{Introduction}

Growing needs for biomedical materials are pushing the limits of biomaterial designs. The primary choices for materials in biomedicine have been metals, ceramics and synthetic polymers, mostly petroleum derived, due to superior mechanical properties, relatively low cost, ease of processing, and availability. These materials are being gradually replaced by degradable synthetic polymers and by biodegradable natural biopolymers (e.g., silk, elastin, collagen, gelatin, alginate, cellulose, keratin, chitin, polyhydroxyalkanoates). This transition follows trends in biomedical materials from static to multifunctional dynamic systems to better address tissue and organ repair, drug and gene delivery, nanotechnology-based imaging, diagnostic platforms and related needs [1,2]. Natural biopolymers offer design flexibility since the composition, structure and degradability can be tailored. Genetic engineering has been used to optimize material properties for specific applications via the incorporation of natural or artificial genes encoding the protein of interest into plasmid DNA. Subsequently this plasmid-gene construct is introduced in a host, most often Escherichia coli [3]. The expression of the artificial gene results in the production of the desired recombinant protein, which is ultimately extracted from the host and purified (Figure 1). Genetic engineering has been utilized to produce recombinant fibrous proteins [3-7], with spider silks as one area of focus due to the remarkable material properties (e.g., high tensile strength, elasticity) $[8,9]$. Collagen fibers are also an outstanding group of fibrous proteins, supporting physiological functions throughout the human body based on combinations of sequence chemistry, different combinations of chains and complex structural hierarchy to achieve both biological and mechanical properties [10]. The list of proteins with superior mechanical performance includes resilin and elastin as well [11].

Producing biopolymers via genetic engineering provides a route to tune properties on several levels: i) incorporation of non-natural amino acids, ii) selection of specific domains and their combinatorial design, iii) functionalization of the sequence, iv) hybrid designs, v) production of dynamic stimuli responsive systems (Figure 2). Aside from expanding the set of available materials, recombinant DNA approaches provide insight into sequence-structure-function relationships. By applying a "bottom- 
up" strategy, complex protein systems can be distilled into specific core functional domains to determine patterns by which structure influences function. These core domains can be combined in different ways to build rationally designed biomaterials. Likewise, recombinant DNA-mediated protein blends can be designed to harbor multiple biopolymer components, otherwise not found together in nature. For instance, Silk-Elastin-Like Polypeptides (SELPs) consist of repeating silk and elastin domains to integrate the physical and biological properties of silk and elastin [12-15]. Collagen-silk chimeric proteins represent another example of such hybrid systems [16]. Numerous, silk-based recombinant hybrid proteins have been investigated to bring new properties or functions to silk. Constructs such as silk-collagen, silk-laminin, silk-reflectin, elastin-resilin-collagen [16-18] have been studied and demonstrate the vast combinatorial design options provided by genetic engineering. The above examples only focus on structural protein building blocks to generate these chimeric fibrous proteins. Chimeric fibrous proteins with new features based on biological or physical recognition have also been generated, including cellular recognition through RGD domains, hydroxyapatite binding domains for bone regeneration and others, but these will not be reviewed here.

In addition to sequence alterations, control of processing parameters allows for another level of material design. However, to take the advantage of advances in biotechnology and processing, precise control of molecular mechanisms that govern protein folding is required. Recent computational approaches have proven useful in advancing this understanding. Some of the key domains in silks responsible for mechanical properties have been identified, as well as the influence of hydration level, solvents, ion content, $\mathrm{pH}$ and protein concentration [19]. Studies of fibrous proteins using replica exchange molecular dynamics have yielded results to compare with experimental structure identification methods [19]. Synergistic approaches based on the integration of processing, experimental and modelling data offer a new discovery path toward material designs (Figure 1). 
Herein, we review the advances in recombinant DNA-mediated fibrous protein production and functionalization of biomaterials to address medical needs, with a special focus on hybrid structural protein systems. We discuss the possibility of the rationale design of fibrous proteins to generate smart stimuli-responsive biomaterials for controlled release and tissue engineering applications. Also, we highlight current challenges of recombinant DNA-mediated protein production and pinpoint some of the strategies that might be undertaken to overcome these limitations.

\section{Recombinant Protein Biomaterial Hybrids and Biomedical Applications}

Some of the current trends in biomaterial science are orientated towards biomaterials that mimic the biological and mechanical properties of natural tissues. Proteins are able to confer mechanical properties to tissues and organs, such as elasticity (elastin, resilin) and strength (collagen, silk). Biocompatibility and cellular invasion, proliferation and differentiation are some of the requisites for biomedical utility. The use of the ribosomal machinery of living organisms as a 'factory' for the production of proteins for biomaterials offers a useful approach to these needs. The advantage in tailoring sequence and in combinatorial designs offers a wide range of constructs, with selected examples outlined in Table 1. Several types of recombinant proteins, such as silk-like proteins (SLPs), elastin-like proteins (ELPs), and collagen-like proteins (CLPs), have been reported and found to have self-assembling or stimuli responsive properties [20]. SLPs form $\beta$-sheets and self-assemble into micelles or vesicles, whereas, ELPs exhibit sharp hydrophilic-to-hydrophobic transitions in response to temperature and ionic strength [20]. These properties have been utilized for development of drug and gene delivery systems (Table 1 ) as well as the production of multifunctional nanoparticles by co-assembling the drug, imaging agent and targeting moieties into single particles.

When SLPS and ELPS are combined into SELPs, sophisticated stimuli responsive systems can be engineered. Current trends and biomedical needs require the design of dynamic proteins that can respond to specific environmental stimuli. Genetic engineering provides a useful toolkit for such 
needs. Namely, by fine tuning the genetic sequences, stimuli or multi-stimuli responsive systems can be generated. In the case of SELPs, sequence variants can be produced by modifying " $X$ " amino acid of elastin domain, GXGVP [20]. The diversity of synthesized SELPs can also be increased using highthroughput synthesis and characterization methods [21]. This combinatorial approach based on designing, building and screening genetic libraries, allows rapid progression toward a functional material. Aside from controlling the genetic sequence, material properties can be modulated by controlled variation of process parameters [22]. SELPs have been processed into various biomaterial formats, from nanoparticles, nanofibers, films, thin coatings, to hydrogels and scaffolds $[20,23]$. However, the main advantage that differentiates SELPs from other recombinant polymers is their stimuli-responsive character; response to various environmental triggers including temperature, $\mathrm{pH}$, ionic strength, redox, enzymatic stimuli and electric fields [20]. This predictive stimuli-sensitivity in physiological conditions is of special value for biomedical applications. The tunable material properties allow SELPs to be pursued as promising biomaterials as nanocarriers and injectable drug release systems, among other dynamic material needs. SELP nanoparticles have been prepared using direct self-assembly, gold nanoparticle triggered self-assembly or hydrophobic drug triggered selfassembly $[21,24,25]$. Although, current applications of SELPs are focused on local hyperthermia based therapeutic delivery in tumors or infections, other promising biomedical applications are being developed: i) controlled delivery of small molecules, DNA and genes; ii) tissue engineering; iii) stimuli-responsive thin coatings; iv) biosensors; v) bio-optics and others [20].

Standardization and purity of natural collagens limit their application, thus CLPs have been pursued as biomaterials with key structural and signaling roles [26]. Significant efforts have been made to produce recombinant DNA-based human collagens, however, requirements for post-translational modifications represent a significant obstacle [26]. CLPs found in bacterial cells are promising candidates, as the triple helix can form without post-translational modifications [26]. This process is mediated more by charge, yet stable variants at body temperatures are achievable. These features allow the use of prokaryotic expression systems. Since the bacterially-derived collagens lack known 
biologically active sites, they can be used as blank templates to incorporate other functionalities. The potential of recombinant human collagens for artificial cornea, wound dressings and other biomedical applications has been demonstrated (Table 1), while translational science for the newer bacterial collagens remains less explored [26]. Bacterial collagens have been shown to be nonimmunogenic, non-toxic and non-thrombogenic and their potential as vascular grafts is being investigated [26]. Several human-derived collagen short amino acid sequences have been inserted into bacterial collagen (e.g., sites for binding integrin $\alpha 2 \beta 1$, fibronectin, heparin, collagenases) [26]. The biological activity of functionalized collagens has been combined with the biomaterial properties of the silk in collagen-silk fusion proteins for tissue engineering applications. Collagen supported more rapid cell interactions with silk scaffolds, improved regulation of stem cell growth and differentiation, as well as fostered the formation of extracellular matrices [16]. The silk contributed robust structure/material properties, avoidance of scaffold shrinkage and provides sustained structures to foster oxygen and nutrient transfer and cell support throughout the matrix.

Resilin, an elastomeric protein with remarkable mechanical properties has gained attention in the biomaterial field. The rubber-like proteins, resilin and elastin, that exhibit reversible deformation, high resilience and elasticity, were combined with a collagen-like sequence. This chimeric elastinresilin-collagen like polypeptide exhibited promising self-assembling properties, formed triple helices and generated fibers [18].

Aside from a genetic engineering chimeric approach, different strategies have been explored to produce protein composites post protein synthesis, where at least one of the components was generated via recombinant DNA methods. For example, excimer-laser technology was used for the ablation of collagen lamellae that were subsequently embedded with recombinant elastin to generate collagen-elastin hybrids. These hybrids were suitable as synthetic extracellular matrices that provided mechanical strength and promoted material-cell interactions [27]. Similarly, synthetic collagen fibers were arranged into parallel arrays, embedded within a thin membrane of a recombinant elastin, and rolled into multi-layered tubes for vascular tissue engineering [28]. 


\section{Current Limitations of Recombinant Production}

Much has been accomplished since recombinant polymers were first explored [4,6,7,29-33]. Protein engineering represents a robust technique for materials science to expand the functional diversity of the resulting protein polymeric products. Despite the recent progress in this direction, several key technical issues remain unresolved. These limitations are in particular linked with the restrictions of the production systems. Namely, when new genes are introduced into a host cell, resources involved in their expression have to be redirected. In E. coli these additional demands for nucleotides, tRNAs, ribosomes and RNA polymerase, may lower the fitness of the host, by affecting the growth rate [34]. Thus it is critical to understand the cellular economy related to gene expression, defined as the distribution of limited cellular resources, to rationally design genetic circuits and control protein yields. Here, rapidly evolving strategies in metabolomics, synthetic biology and related fields can have an immense impact.

The expression of highly repetitive genes as in the case of sequences encoding fibrous proteins brings additional hurdles to this challenge. Aside from gene instability and a high frequency of homologous recombination, there are considerable constraints imposed by the bacterial ribosomal machinery. Common problems involve discontinuous translation and low expression levels due to: i) limited tRNA availability leading to early termination and truncation [35,36]; ii) the presence of mRNA secondary structure possibly causing translation pauses and fall-offs at the ribosome [5]; iii) codon preferences and incompatibility with prokaryotic hosts such as E. coli. As previously mentioned, the production of mammalian collagen is limited to eukaryotic systems, as prokaryotic systems and yeast lack the post translational modification machinery required for proper protein folding. These issues in combination with the more common challenge of the formation of insoluble inclusion bodies and modest yields of polymers, limit the cost-effective production and commercialization of recombinant fibrous protein-based biomaterials. With the advent of the newer bacterial collagens, some of the later challenges may be circumvented; however, overall yields remain an issue, particularly when combined with the challenge of recapitulating the 
structural hierarchy from the recombinant collagens. Plant expression systems may aid in these challenges.

Numerous strategies have been explored to improve the production/purification process of recombinant DNA-derived fibrous proteins. The suitability of numerous host cells has been analyzed, and metabolic engineering approaches have been initiated to modify the corresponding pathways $[6,29,37-40]$. Recent breakthroughs in synthetic biology allowed the construction of novel networks and pathways to rewire and reprogram host cells [41] with the ultimate goal to obtain superior secretion of recombinant DNA-derived fibrous proteins [42]. Understanding the interactions arising in natural systems is also of critical importance for the rational design of synthetic circuits and to establish a predictive framework to determine how circuit behavior is affected by the competition for limited cellular resources. Mathematical models are starting to bring significant improvement to this need, however their application for fibrous protein production is just beginning to have impact [34].

\section{Conclusions}

The need for novel and tailored biomaterials continues to grow in importance, for example, to direct cell and tissue responses, to match mechanical properties and to match degradation rate or turnover in vivo to the rate of new tissue growth. Genetic engineering provides new avenues for biopolymer designs and this approach has yielded new families of biopolymers to match specific material functions. These new designs can be used to generate tailored biopolymer designs and dynamic stimuli-responsive materials. Insights into sequence-function relationships have been gained through combinatorial-screening approaches to also provide a guide to future polymer designs. Recombinant DNA technology facilitates the production of fibrous proteins, however highly repetitive sequences encoding these proteins and the limitations of expression, yield and size remain as major challenges. The development of new strategies from synthetic/systems biology should offer major help in these directions. 


\section{References and recommended reading}

Papers of particular interest, published within the period of review, have been highlighted as:

- of special interest

•• of outstanding interest

\section{Acknowledgement}

We thank the NIH (P41 EB002520), the AFOSR and the NSF IGERT program for support of this work.

\section{References}

1. Hench LL: Biomaterials: A forecast for the future. Biomaterials 1998, 19:1419-1423.

2. Williams DF: On the nature of biomaterials. Biomaterials 2009, 30:5897-5909.

3. - Tokareva O, Michalczechen-Lacerda V, Rech EL, Kaplan DL: Recombinant DNA production of spider silk proteins. Microb. Biotechnol. 2013, 6:651-663.

3. - The authors review recombinant DNA techniques currently used for cloning of spider silk-like sequences and hosts used for their expression. They also highlight the limitations of production system.

4. Bedell-Hogan D, Trackman P, Abrams W, Rosenbloom J, Kagan H: Oxidation, cross-linking, and insolubilization of recombinant tropoelastin by purified lysyl oxidase. J. Biol. Chem. 1993, 268:10345-10350.

5. Fahnestock SR, Irwin SL: Synthetic spider dragline silk proteins and their production in Escherichia coli. Appl. Microbiol. Biotechnol. 1997, 47:23-32.

6. Prince JT, Mcgrath KP, Digirolamo JCM, Kaplan DL: Construction, Cloning, and Expression of Synthetic Genes Encoding Spider Dragline Silk. Biochemistry 1995, 34:10879-10885.

7. Rabotyagova OS, Cebe $P$, Kaplan DL: Self-assembly of genetically engineered spider silk block copolymers. Biomacromolecules 2009, 10:229-236.

8. Gosline JM, Guerette PA, Ortlepp CS, Savage KN: The mechanical design of spider silk: From fibroin sequence to mechanical function. J. Exp. Biol. 1999, 3303:3295-3303.

9. Tillinghast EK, Kavanagh EJ: The alkaline proteases of Argiope and their possible role in web digestion. J. Exp. Zool. 1977, 202:2113-2222.

10. Wong Po Foo C, Kaplan DL: Genetic engineering of fibrous proteins: spider dragline silk and collagen. Adv. Drug Deliv. Rev. 2002, 54:1131-1143. 
11. •-Rodríguez-Cabello JC, Prieto S, Arias F, Reguera J, Ribeiro A: Nanobiotechnological approach to engineered biomaterial design: the example of elastin-like polymers. Nanomedicine (Lond). 2006, 1:267-280.

11. - This article provides arguments concerning the role of biotechnology and nanotechnology in the future development of advanced biomaterial. Also it discusses the potential of these biomaterials for the development of biocompatible biofunctional platforms.

12. Hu X, Wang X, Rnjak J, Weiss AS, Kaplan DL: Biomaterials derived from silk-tropoelastin protein systems. Biomaterials 2010, 31:8121-8131.

13. Machado R, Azevedo-Silva J, Correia C, Collins T, Arias FJ, Rodríguez-Cabello JC, Casal M: High level expression and facile purification of recombinant silk-elastin-like polymers in auto induction shake flask cultures. AMB Express 2013, 3:11.

14. Collins T, Azevedo-Silva J, da Costa A, Branca F, Machado R, Casal M: Batch production of a silk-elastin-like protein in E. coli BL21(DE3): key parameters for optimisation. Microb. Cell Fact. 2013, 12:21.

15. Megeed Z, Cappello J, Ghandehari H: Genetically engineered silk-elastinlike protein polymers for controlled drug delivery. Adv. Drug Deliv. Rev. 2002, 54:1075-1091.

16. An B, DesRochers T, Qin G, Xia X-X, Thiagarajan G, Brodsky B, Kaplan DL: The Influence of Specific Binding of Collagen-Silk Chimeras to Silk Biomaterials on hMSC Behavior. Biomaterials 2013, 34:402-412.

17. Hossainy S, Ngo M, Trollsas M, Stankus J, Gene M: Methods and compositions for treating tissue using silk proteins. 2012, US 20130251764 A1.

18. - Bracalello A, Santopietro V, Vassalli M, Marletta G, Del Gaudio R, Bochicchio B, Pepe A: Design and production of a chimeric resilin-, elastin-, and collagen-like engineered polypeptide. Biomacromolecules 2011, 12:2957-2965.

18. - This study describes the potential of genetic engineering for production of new proteins that do not exist in nature by combining sequences coding for different proteins.

19. Gronau G, Krishnaji S, Kinahan M, Giesa T, Wong J, Kaplan DL, Buehler M: A review of combined experimental and computational procedures for assessing biopolymer structureprocess-property relationships. Biomaterials 2012, 33:8240-8255.

20. • Hunag W, Rollett A, Kaplan DL: Silk-elastin-like protein biomaterials for the controlled delivery of therapeutics. 2015, 12:779-791.

20. $\bullet$ This review describes the potential of SELP proteins as dynamic system, where controlled delivery can be achieved by applying different environmental stimuli.

21. Wang $Q$, Xia X, Huang W, Lin $Y, X u$ Q, Kaplan DL: High Throughput Screening of Dynamic SilkElastin-Like Protein Biomaterials. Adv. Funct. Mater. 2014, 24:4303-4310.

22. •- Spiess K, Lammel A, Scheibel T: Recombinant spider silk proteins for applications in biomaterials. Macromol. Biosci. 2010, 10:998-1007. 
22. - The authors provide all encompassing data on potential application of recombinant spider silk for biomedical purposes.

23. Qiu W, Huang Y, Teng W, Cohn CM, Cappello J, Wu X: Complete recombinant silk-elastinlike protein-based tissue scaffold. Biomacromolecules 2010, 11:3219-2327.

24. - Xia X-X, Xu Q, Hu X, Qin G, Kaplan DL: Tunable self-assembly of genetically engineered silk-elastin-like protein polymers. Biomacromolecules 2011, 12:3844-3850.

24. - Fundamentals of self-assembly characteristics of silk-elastin like protein polymers and its potential use for controlled drug delivery and biomedical engineering.

25. Lin Y, Xia X, Wang M, Wang Q, An B, Tao H, Xu Q, Omenetto F, Kaplan DL: Genetically programmable thermoresponsive plasmonic gold/silk-elastin protein core/shell nanoparticles. Langmuir 2014, 30:4406-4414.

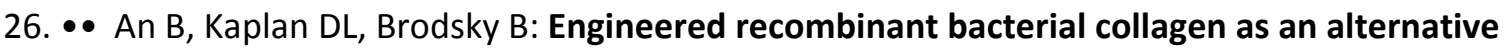
collagen-based biomaterial for tissue engineering. Front. Chem. 2014, 2:1-5.

26. $\bullet$ This review describes the potential of bacterial collagen as a blank template for integration of functionalities and highlights its advantages over mammalian collagen.

27. Kumar V, Martinez AW, Caves JM, Naik N, Haller C, Chaikof EL: Microablation of collagenbased substrates for soft tissue engineering. Biomed. Mater. 2014, 9:1-8.

28. Caves JM, Kumar V, Martinez AW, Kim J, Ripberger CM, Haller C, Chaikof EL: The use of microfiber composites of elastin-like protein matrix reinforced with synthetic collagen in the design of vascular grafts. Biomaterials 2010, 31:7175-7182.

29. Fahnestock SR, Bedzyk L: Production of synthetic spider dragline silk protein in Pichia pastoris. Appl. Microbiol. Biotechnol. 1997, 47:33-39.

30. Xu L, Rainey JK, Meng Q, Liu X-Q: Recombinant minimalist spider wrapping silk proteins capable of native-like fiber formation. PLoS One 2012, 7:e50227.

31. Sobel ME, Yamamoto T, Adams SL, Dilauro R, Enrico V, Crombrugghe BDE, Pastan IRA: Construction of a recombinant bacterial plasmid containing a chick pro-a2 collagen gene sequence. 1978, 75:5846-5850.

32. Fazio MJ, Olsen DR, Kauh EA, Baldwin CT, Indik Z, Goldstein N, Yeh H, Rosenbloom J, Uitto J: Cloning of Full-length Elastin cDNAs from a Human Skin Fibroblast Recombinant cDNA Library: Further Elucidation of Alternative Splicing Utilization Exon-specific Oligonucleotides. J. Invest. Dermatol. 1988, 91:458-464.

33. Lehrach H, Maria A, Hanahan D, Wozney J, Fuller F, Crkvenjakov R, Boedtker H, Doty P: Construction and characterization of a 2.5 Kilobase Procollagen Clone. Proc. Natl. Acad. Sci. 1978, 75:5417-5421.

34. •- Gyorgy A, Jiménez Jl, Yazbek J, Huang H-H, Chung H, Weiss R, Del Vecchio D: Isocost Lines Describe the Cellular Economy of Genetic Circuits. Biophys. J. 2015, 109:639-646. 
34. - Predicting behavior of a genetic circuits composed of multiple modules that share resources to provide design guidelines for minimizing the effects of such couplings.

35. Candelas G, Arroyo G, Carrasco C, Dompenciel R: Spider silk glands contain a tissue-specific alanine tRNA that accumulates in vitro in response to the stimulus for silk protein synthesis. Dev. Biol. 1990, 140:215-220.

36. Martin SL, Vrhovski B, Weiss AS: Total synthesis and expression in Escherichia coli of a gene encoding human tropoelastin. 1995, 154:159-166.

37. Huemmerich D, Scheibel T, Vollrath F, Cohen S, Gat U, Ittah S: Novel Assembly Properties of Recombinant Spider Dragline Silk Proteins. Curr. Biol. 2004, 14:2070-2074.

38. Lazaris A, Arcidiacono S, Huang Y, Zhou J-F, Duguay F, Chretien N, Welsh E, Soares JW, Karatzas $\mathrm{CN}$ : Spider silk fibers spun from soluble recombinant silk produced in mammalian cells. Science 2002, 295:472-476.

39. Scheller J, Guhrs K, Grosse F, Conrad U, Gührs K: Production of Spider Silk Proteins in Tobacco and Potato. Nat. Biotechnol. 2001, 19:573-577.

40. - Xia X-X, Qian Z-G, Ki CS, Park YH, Kaplan DL, Lee SY: Native-sized recombinant spider silk protein produced in metabolically engineered Escherichia coli results in a strong fiber. Proc. Natl. Acad. Sci. U. S. A. 2010, 107:14059-14063.

40. - Potential of metabolic engineering to re-direct bacterial pathways toward the production of full-length spider silk.

41. • Khalil A, Collins J: Synthetic biology: applications come of age. Nat. Rev. Genet. 2010, 11:367-379.

41. - Synthetic biology has been used to rewire and reprogram organisms. This will lead to cheaper drugs, targeted therapies for attacking 'superbugs' and diseases, such as cancer.

42. Widmaier DM, Tullman-Ercek D, Mirsky E, Hill R, Govindarajan S, Minshull J, Voigt C: Engineering the Salmonella type III secretion system to export spider silk monomers. Mol. Syst. Biol. 2009, 5:309.

43. Tokareva OS, Glettig DL, Abbott RD, Kaplan DL: Multifunctional spider silk polymers for gene delivery to human mesenchymal stem cells. J. Biomed. Mater. Res. Part B Appl. Biomater. 2014, 103:1390-1401.

44. Huang J, Wong C, George A, Kaplan DL: The effect of genetically engineered spider silkdentin matrix protein 1 chimeric protein on hydroxyapatite nucleation. Biomaterials 2007, 28:2358-2367.

45. - Gomes S, Leonor IB, Mano JF, Reis RL, Kaplan DL: Natural and genetically engineered proteins for tissue engineering. Prog. Polym. Sci. 2012, 37:1-17.

45. - This review address some of the most frequently used recombinant proteins for tissue engineering and biomedical applications and describes the techniques most commonly used 
to functionalize protein-based biomaterials by combining them with bioactive molecules to enhance biological performance.

46. Wong Po Foo C, Patwardhan S V, Belton DJ, Kitchel B, Anastasiades D, Huang J, Naik RR, Perry $\mathrm{CC}$, Kaplan DL: Novel nanocomposites from spider silk-silica fusion (chimeric) proteins. Proc. Natl. Acad. Sci. U. S. A. 2006, 103:9428-9433.

47. Floss DM, Schallau K, Rose-John S, Conrad U, Scheller J: Elastin-like polypeptides revolutionize recombinant protein expression and their biomedical application. Trends Biotechnol. 2010, 28:37-45.

48. MacKay JA, Chen M, McDaniel JR, Liu W, Simnick AJ, Chilkoti A: Self-assembling chimeric polypeptide-doxorubicin conjugate nanoparticles that abolish tumours after a single injection. Nat. Mater. 2009, 8:993-999.

49. McDaniel JR, Bhattacharyya J, Vargo KB, Hassouneh W, Hammer D, Chilkoti A: Self-assembly of thermally responsive nanoparticles of a genetically encoded peptide polymer by drug conjugation. Angew. Chemie - Int. Ed. 2013, 52:1683-1687. 
Graphical Abstract. Historical overview of biomaterial development through the prism of genetic engineering. Recombinant DNA-based fibrous protein production has undergone significant upgrades. Sequence-structure-function have been analyzed to understand how to better design biomaterials with more tunable features. Metabolic engineering has also been utilized to improve the production process and different combinatorial designs have been investigated to generate dynamic biomaterial systems.

Table 1. Recombinant DNA-derived fibrous proteins chimeras and their potential biomedical applications.

Figure 1. Integrated modelling and experimental approaches in biomaterials designs. Recombinant DNA-derived fibrous protein production with key steps shown such as cloning, expression of designed genetic construct and target protein purification can be combined with the control of processing parameters and computational modelling to develop new platforms for biomaterials.

Figure 2. Expanding the potential of biomaterial designs via genetic engineering. Selected strategies include incorporation of non-natural amino acids, selection of specific domains and their combinatorial design, functionalization of the sequence, recombinant protein chimeric designs, and production of dynamic stimuli responsive systems. 
Table 1.

\begin{tabular}{|c|c|c|c|}
\hline \multicolumn{2}{|r|}{ Recombinant Protein Blend } & Applications & References \\
\hline \multirow{7}{*}{ 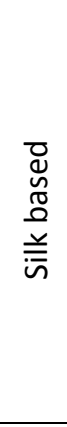 } & Spider silk-NLS-HAB-TLM & Gene delivery & [43] \\
\hline & Spider silk-dentin matrix protein & Tissue engineering/Biomedical & [44] \\
\hline & Spider silk-bone sialoprotein & Biomedical/Tissue engineering & [45] \\
\hline & Spider silk-biomineralization peptides & Bone regeneration & [46] \\
\hline & Spider silk-antimicrobial peptides & Biomedical/Tissue engineering & [45] \\
\hline & Bombyx mori silk-RGD-elastin & Biomedical applications & [45] \\
\hline & RGD-silk fibroin & Chondrogenesis enhancement & [45] \\
\hline \multirow{6}{*}{ 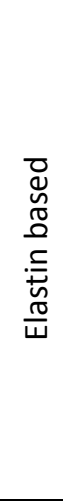 } & $\begin{array}{l}\text { ELP-cell penetrating peptide-cell cycle inhibitor } \\
\text { peptide }\end{array}$ & Drug delivery/cancer therapy & [47] \\
\hline & ELPs-doxorubicin & Biomedical/Cancer therapy & {$[48,49]$} \\
\hline & ELP-RGD & Tissue Engineering & [47] \\
\hline & ELP-TNFR & Delivery of anti-TNF drugs & [47] \\
\hline & Silk-elastin-like polymers & $\begin{array}{l}\text { Tissue Engineering } \\
\text { /Nanomedicine }\end{array}$ & {$[20,23]$} \\
\hline & Au-SELP & Plasmonic nanodevice & [25] \\
\hline \multirow{9}{*}{ 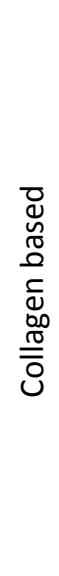 } & BMP-2 - collagen-biding domain & Bone repair & [45] \\
\hline & $\begin{array}{l}\text { Scl2 Bacterial collagen like domain-fibronectin binding } \\
\text { site-silk }\end{array}$ & Biomedical/Bone regeneration & [16] \\
\hline & Scl2 Bacterial collagen like domain-integrin binding site & Biomedical/Tissue engineering & [26] \\
\hline & Scl2 Bacterial collagen like domain-heparin binding site & Biomedical/Tissue engineering & [26] \\
\hline & Scl2 Bacterial collagen like domain-MMP binding site & Biomedical/Tissue engineering & [26] \\
\hline & TGF-B1-F1/TGF-B1-F2 - collagen binding domain & Biomedical/Tissue engineering & [45] \\
\hline & FGF/FGF-F1/FGF-F2-collagen binding domain & Delivery systems/Biomedical & [45] \\
\hline & VEGF/PDGF-collagen binding domain & Wound repair & [45] \\
\hline & NGF- $\beta$-collagen binding domain & Delivery system & [45] \\
\hline
\end{tabular}

NLS - nuclear localization sequence; HAB - human mesenchymal stem cell high affinity binding peptide; TLM translocation motif; RGD = arginine, glycine, aspartic acid; TNFR - tumor necrosis factor receptor; TNF - tumor necrosis factor; BMP - bone morphogenetic protein; SIc2 - Streptococcal collagen-like protein 2; MMP - matrix metalloproteinase; TGF - transforming growth factor; TGF-B1-F1/TGF-B1-F2 - fusion proteins consisting of a purification tag, a protease-sensitive linker/collagen binding domain, and a CDNA sequence encoding the active fragment of human TGF-beta 1; FGF - fibroblast growth factor; VEGF - vascular endothelial growth factor; PDGF - platelet-derived growth factor; NGF - nerve growth factor. 


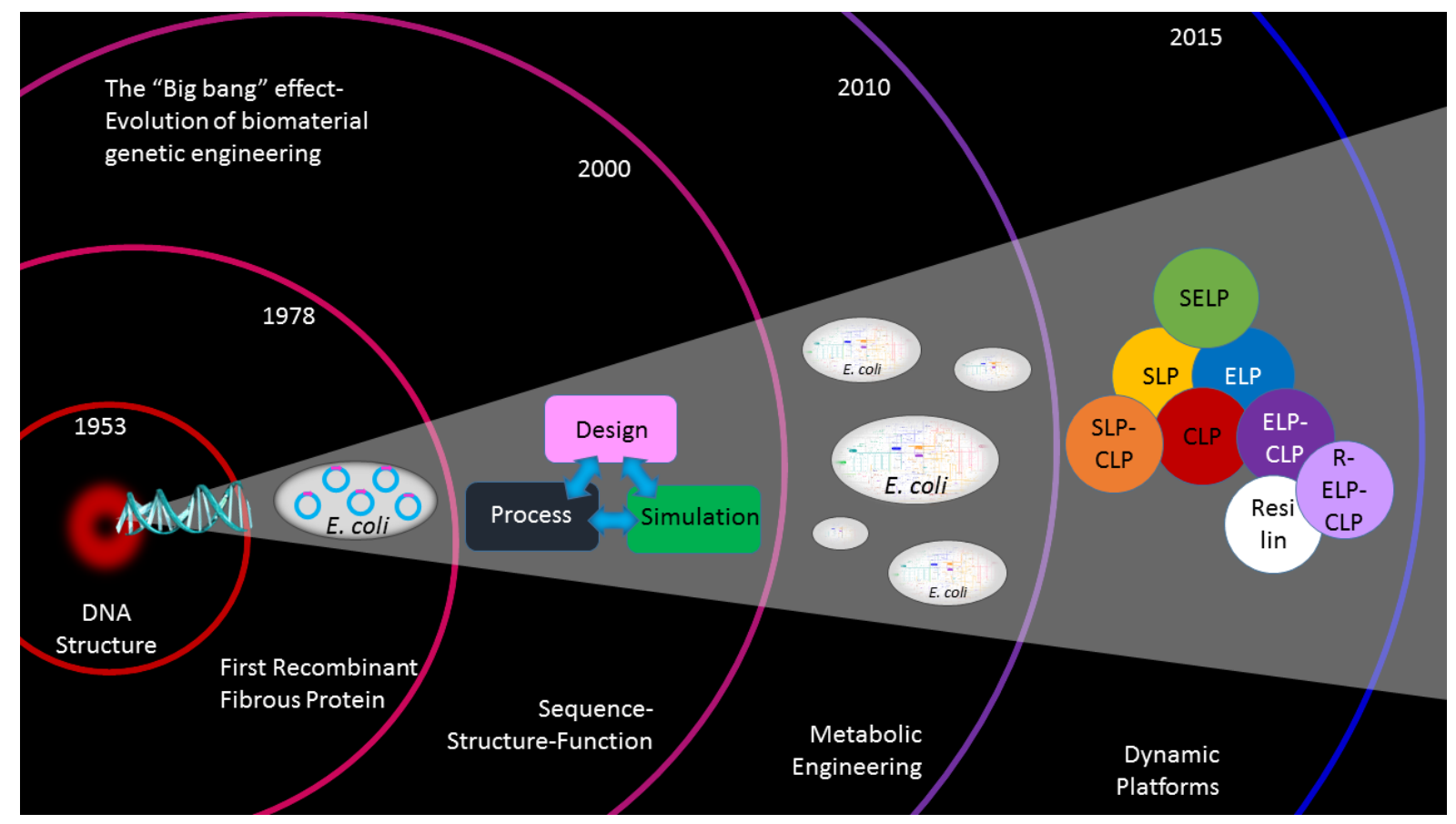

Graphical Abstract. Dinjaski and Kaplan, 2015 


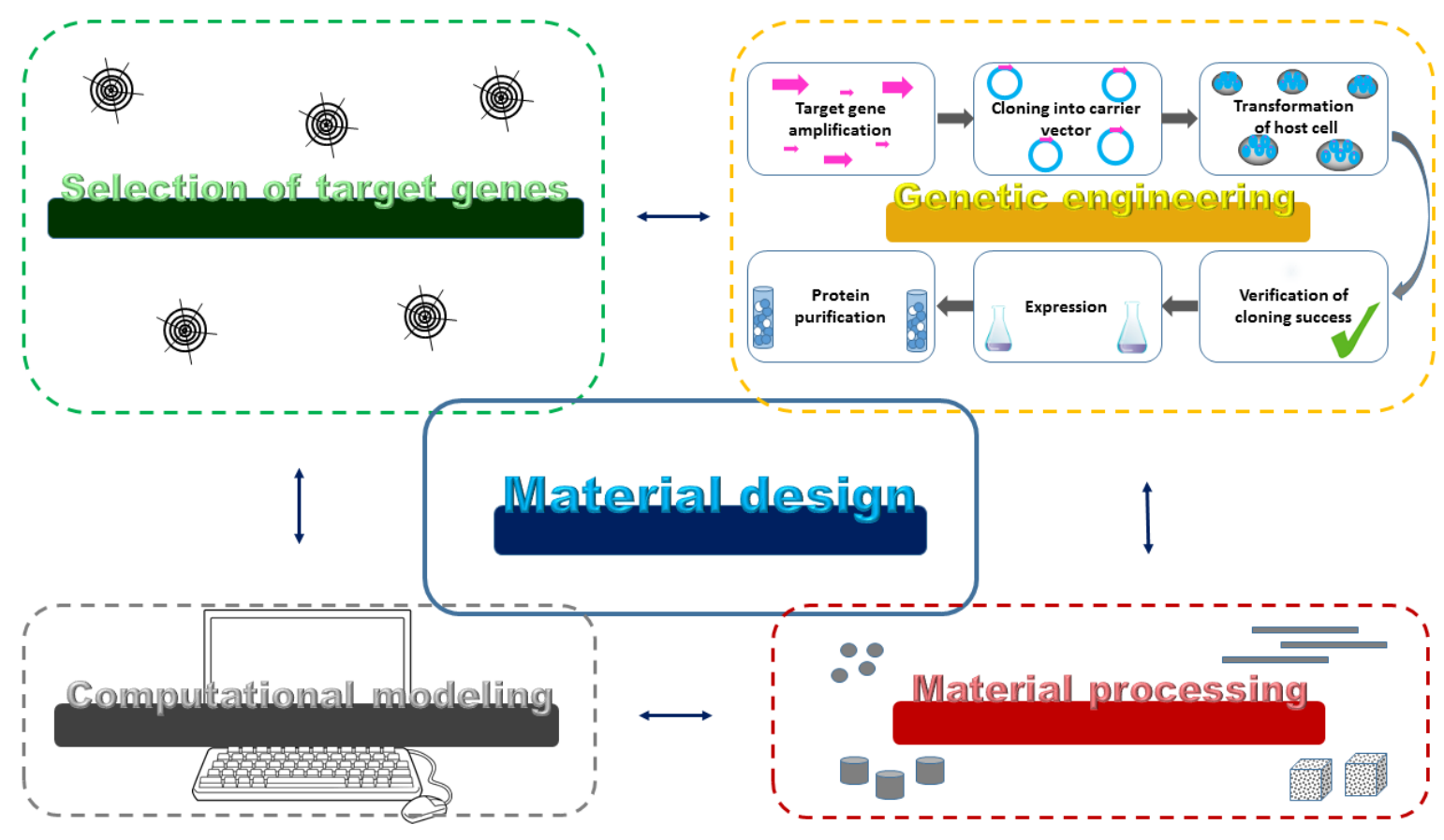

Figure 1. Dinjaski and Kaplan, 2015 


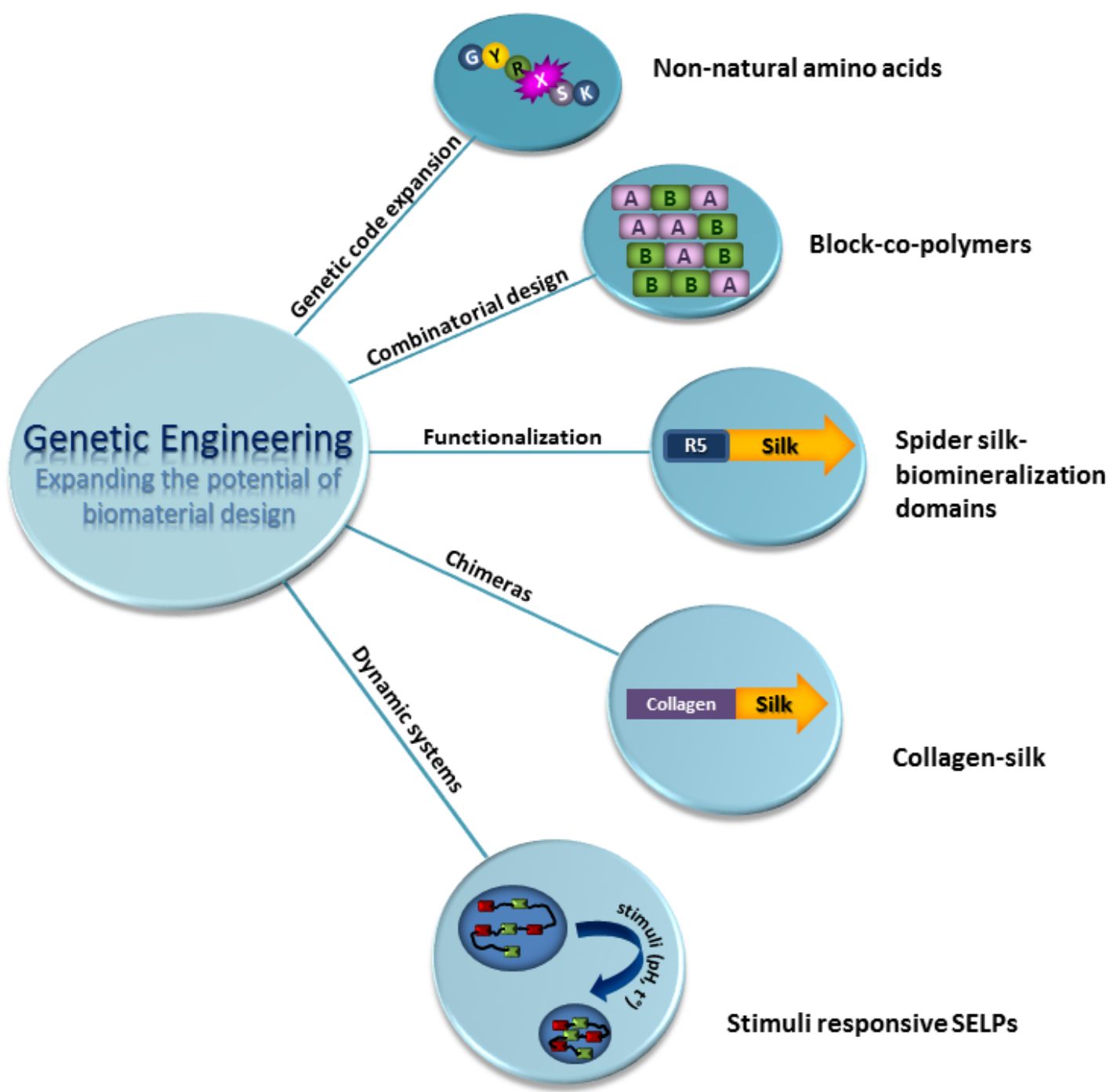

Figure 2. Dinjaski and Kaplan, 2015 